\title{
Brachytherapy for localized prostate cancer in the modern era: a comparison of patient-reported quality of life outcomes among different techniques
}

\author{
Tiffany M. Morgan, MD ${ }^{1,4}$, Robert H. Press, MD ${ }^{1,4}$, Patrick K. Cutrell, MS',4, Chao Zhang, PhD2,4, Zhengja Chen, PhD 2,4, \\ Sara Rahnema, MS, DABR ${ }^{1,4}$, Martin Sanda, MD 3,4, John Pattaras, MD³, , Pretesh R. Patel, MD',4, Ashesh B. Jani, MDl,4, \\ Peter J. Rossi, MDl,4 \\ 'Department of Radiation Oncology, ${ }^{2}$ Department of Biostatistics and Bioinformatics, ${ }^{3}$ Department of Urology, ${ }^{4}$ Winship Cancer Institute, \\ Emory University. Atlanta, GA USA
}

\begin{abstract}
Purpose: To report our institutional quality of life (QOL) data for low-dose-rate (LDR) monotherapy (LDR mono), high-dose-rate (HDR) monotherapy (HDR mono), and EBRT with an HDR brachytherapy boost (HDR boost).

Material and methods: The charts of 165 patients with localized adenocarcinoma of the prostate treated with LDR monotherapy (LDR mono), HDR monotherapy (HDR mono), and EBRT with an HDR brachytherapy boost (HDR boost) at a single institution between 2012 and 2015 were reviewed. All patients completed the American Urological Association symptom score (AUASS) and Expanded Prostate Index for Prostate Cancer - Clinical Practice (EPIC$\mathrm{CP})$ quality of life assessments prior to treatment and at least one follow-up survey. Time points included baseline, $\leq 2$ months, $2-\leq 6$ months, $6-\leq 12$ months, $12-\leq 18$ months, 18- $\leq 24$ months, $24-\leq 30$ months, and $>30$ months. Linear mixed models were performed to test for significant changes and differences in each outcome over time.

Results: Mean follow-up was 19.5 months. All major functional QOL domains were affected after treatment with brachytherapy for localized prostate cancer. All domains improved over time, with the exception of sexual function scores for all groups and urinary incontinence scores for the HDR mono group. Patients treated with LDR did have higher AUA, irritability/obstructive symptoms, incontinence, bowel, and QOL scores acutely compared to the HDR and HDR + boost groups. Vitality scores were significantly worse in the HDR boost group both acutely and at the $>$ 30-month time point.

Conclusions: Patients receiving HDR brachytherapy had lower acute urinary and rectal toxicity compared to the patients receiving LDR, even when combined with EBRT. However, long-term toxicity was similar.

Key words: brachytherapy, quality of life, prostatic neoplasms.

\section{Purpose}

Prostate cancer remains a major health concern in the United States, with an estimated 161,360 diagnoses in 2017 alone [1]. However, in the era of prostate-specific antigen (PSA) surveillance, a majority of men have localized disease at presentation [2,3]. In this modern era, there are many management strategies available to those with localized disease, including active surveillance, radical prostatectomy, and radiation therapy. With high survival rates associated with each of these techniques, men and their partners often make a treatment decision based on their understanding of quality of life (QOL) differences between each treatment modality [4]. There have been multiple prospective studies assessing patient-reported toxicity differences among the 3 major definitive therapy options: prostatectomy, external beam radiation therapy (EBRT), and brachytherapy $[5,6]$.

Regarding radiation therapy, dose escalated EBRT has been found to improve clinical outcomes in multiple randomized trials $[7,8,9,10,11]$. However, increasing doses of EBRT may be associated with increased toxicity [12]. While biochemical and toxicity outcomes appear similar with extreme hypofractionation via stereotactic body radiation therapy (SBRT) compared to conventional techniques, long-term follow-up is lacking $[13,14,15]$. Given the rapid dose fall off associated with brachytherapy, one can achieve an increased BED while limiting normal tissue toxicity. Both high-dose-rate (HDR) and low-doserate (LDR) techniques exist, each with a unique set of ad-
Address for correspondence: Tiffany M. Morgan, MD, Department of Radiation Oncology, The Winship Cancer Institute of Emory University, 1365 Clifton Road NE, Room C-5092, Atlanta, GA 30322, USA, phone: +1 404778-3473, fax: +1 404 778-4139, ø e-mail: tiffany.morgan2@emory.edu
Received: 08.05 .2018

Accepted: 10.09 .2018

Published: 28.12 .2018 
vantages and disadvantages $[16,17,18]$. In the definitive management of prostate cancer, brachytherapy can be used as a monotherapy or as a boost with EBRT depending on the aggressiveness of the disease, with favorable outcomes compared to EBRT alone $[19,20]$. With equivalent oncologic outcomes between HDR and LDR techniques, quality of life differences between the two become paramount. It is also important for patients to understand what increases in toxicity they are accepting, when brachytherapy is added as a boost after EBRT [21,22]. We report our institutional QOL data for LDR monotherapy (LDR mono), HDR monotherapy (HDR mono), and EBRT with an HDR brachytherapy boost (HDR boost).

\section{Material and methods}

After institutional review board approval, the charts of 202 patients with biopsy proven and clinically localized adenocarcinoma of the prostate, treated with HDR or LDR brachytherapy between June 2012 and December 2015, were reviewed. Patients were treated either with HDR mono, HDR boost, or LDR mono radiotherapy (RT). Patient-reported outcomes (PRO) were collected at baseline and at regular intervals after treatment using the American Urological Association symptom score (AUASS) and the Expanded Prostate Index for Prostate Cancer - Clinical Practice (EPIC-CP) [23,24]. The American Urological Association developed the AUASS to determine how bothersome men's urinary symptoms are. Each urinary symptom is given a score from 0-5. A score of 0 means the symptoms do not occur at all, while a score of 3 means men experience the symptoms about half the time. A score of 5 means the symptoms occur almost always. The AUASS consists of 7 urinary specific questions and is scored out of a total of 35 points. The EPIC-CP is divided into subdomains, including urinary incontinence (incon), urinary irritability and obstructive symptoms (irr/obs), bowel function, sexual function, and vitality. Each subdomain consists of 3 questions and is scored out of a total of 12 points, while the overall EPIC-CP quality of life score is out of a total of 60 points. Patients without baseline surveys and at least one follow-up survey, and patients who were previously treated for prostate cancer were excluded, leaving 165 patients for review.

\section{Brachytherapy treatment}

For both HDR and LDR brachytherapy, biplanar ultrasonography was performed in transverse and sagittal dimensions to identify the prostate, seminal vesicles, bladder, and rectum (Hitachi Hi VISION Avius, Tokyo, Japan). For HDR, a transperineal interstitial brachytherapy template was used under ultrasound guidance to place 5 or 6-French HDR catheters into the prostate at the appropriate depth circumferentially around the peripheral capsule of the prostate, avoiding the urethra. Computed tomography (CT) simulation was then performed to verify brachytherapy catheter depth and for treatment planning. The treatment plan was generated with comprehensive treatment planning software (Oncentra Brachy, Stockholm, Sweden). Treatment was delivered to the target volume using a remote after loaded Ir-192 source. When delivered as monotherapy, HDR dose was 13.5 Gy in 2 fractions $(n=73)$ or 12 Gy in 2 fractions $(n=2)$. When delivered as a boost after EBRT, the HDR dose was $9.5 \mathrm{~Gy} \times 2$ fractions $(n=38), 10 \mathrm{~Gy} \times 2$ fractions $(n=10), 10.5 \times 2$ fractions $(n=3)$, or $15 \mathrm{~Gy} \times 1$ fraction $(n=4)$. The doses used represent the evolution of our practice to a higher dose per fraction, with the 2-fraction regimen for both monotherapy and boost. For boost patients, a single fraction regimen was ultimately used for patient convenience $[25,26,27,28,29]$. For LDR, a template grid was also applied, and using the previously configured plan, palladium-103 seeds were placed under ultrasound guidance using pre-loaded needles. Following review of the real-time ultrasound dosimetry, a decision was made whether or not to supplement the preplanned seed configuration. For all LDR monotherapy patients, the prescribed dose was $125 \mathrm{~Gy}$.

\section{EBRT treatment}

All patients in the HDR boost group received EBRT prior to brachytherapy. The clinical target volume (CTV) standardly consisted of the prostate and seminal vesicles, and a $0.3-1 \mathrm{~cm}$ margin was added to the CTV to create the planning target volume (PTV). Treatment doses included 45 Gy in 25 fractions $(n=38), 37.5$ Gy in 15 fractions $(n=14)$, and other $(n=3)$. All patients were treated with either intensity-modulated radiation therapy (IMRT) or volumetric-modulated arc radiotherapy (VMAT) under daily image guidance. Treatment details for brachytherapy and EBRT are listed in Table 1.

\section{Statistical analysis}

Three groups of prostate cancer patients treated with 3 regimens (HDR mono, HDR boost, and LDR mono) were included in the analysis. Outcomes include AUA score and EPIC-CP subdomain scores - incon, irr/obs, bowel, sexual function, vitality, and QOL. All outcome variables were treated as the continuous variables. For descriptive statistics of each outcome, time points were set-up as baseline, $\leq 2$ months, $2-\leq 6$ months, $6-\leq 12$ months, $12-\leq 18$ months, $18-\leq 24$ months, $24-\leq 30$ months, and $>30$ months based on standard post-treatment follow-up visit times in our department. The mean and standard deviation of the outcomes of the 3 different treatment groups at different time points were calculated. Linear mixed models were performed to test whether there were any significant changes over time for each outcome and to detect whether there were any significant differences of each outcome among the different treatment groups. The significance level was set at 0.05. SAS 9.4 was used for data analyses and management.

\section{Results}

\section{Patient characteristics}

A total of 165 men with localized prostate cancer were included in the study. Thirty five patients underwent treatment with LDR mono, 75 with HDR mono, and 55 with an HDR boost. Median follow-up for all groups 
Table 1. Patient and treatment characteristics

\begin{tabular}{|c|c|c|c|c|c|}
\hline & $\begin{array}{l}\text { LDR monotherapy } \\
(n=35)\end{array}$ & $\begin{array}{l}\text { HDR monotherapy } \\
(n=75)\end{array}$ & $\begin{array}{c}\text { EBRT }+ \text { HDR boost } \\
(n=55)\end{array}$ & $\begin{array}{c}\text { All } \\
(n=165)\end{array}$ & $p$ value \\
\hline Median follow-up (months) & $14(1.1-49.6)$ & $18.7(1.1-52)$ & $23(1.6-50.6)$ & $19.47(1.1-51.97)$ & \\
\hline $\begin{array}{l}\text { Median age at treatment } \\
\text { (years) }\end{array}$ & $65(50-75)$ & $65(48-87)$ & $68(55-82)$ & $66(48-87)$ & 0.011 \\
\hline Median prostate volume (cc) & $33.6(15.13-62.7)$ & $49.05(22.69-96.13)$ & $41.23(21.58-84.1)$ & $41.5(15.1-96.13)$ & $<0.001$ \\
\hline T stage & & & & & 0.020 \\
\hline $\mathrm{T} 1 \mathrm{~b}$ & 0 & 1 & 0 & 1 & \\
\hline T1c & 33 & 63 & 36 & 132 & \\
\hline $\mathrm{T} 2 \mathrm{a}$ & 2 & 10 & 9 & 21 & \\
\hline $\mathrm{T} 2 \mathrm{~b}$ & 0 & 1 & 2 & 3 & \\
\hline $\mathrm{T} 2 \mathrm{c}$ & 0 & 0 & 3 & 3 & \\
\hline T3a & 0 & 0 & 3 & 3 & \\
\hline $\mathrm{T} 3 \mathrm{~b}$ & 0 & 0 & 2 & 2 & \\
\hline \multicolumn{6}{|l|}{ Gleason score } \\
\hline $3+3$ & 17 & 26 & 0 & 43 & $<0.001$ \\
\hline $3+4$ & 18 & 45 & 23 & 86 & \\
\hline $4+3$ & 0 & 3 & 13 & 16 & \\
\hline $4+4$ & 0 & 1 & 10 & 11 & \\
\hline $4+5$ & 0 & 0 & 5 & 5 & \\
\hline $5+4$ & 0 & 0 & 3 & 3 & \\
\hline $5+5$ & 0 & 0 & 1 & 1 & \\
\hline PSA & & & & & 0.004 \\
\hline$<10$ & 30 & 71 & 40 & 141 & \\
\hline $10-20$ & 5 & 3 & 12 & 20 & \\
\hline$>20$ & 0 & 1 & 3 & 4 & \\
\hline Brachytherapy dose (Gy) & & & & & $<0.001$ \\
\hline $9.5 \mathrm{~Gy} \times 2$ fractions & 0 & 0 & 38 & 38 & \\
\hline 10 Gy $\times 2$ fractions & 0 & 0 & 10 & 10 & \\
\hline 10.5 Gy $\times 2$ fractions & 0 & 0 & 3 & 3 & \\
\hline 12 Gy $\times 2$ fractions & 0 & 2 & 0 & 2 & \\
\hline 13.5 Gy $\times 2$ fractions & 0 & 73 & 0 & 73 & \\
\hline 15 Gy × 1 fraction & 0 & 0 & 4 & 4 & \\
\hline 125 Gy & 35 & 0 & 0 & 35 & \\
\hline EBRT dose & & & & & $<0.001$ \\
\hline 45 Gy in 25 fractions & - & - & 38 & 38 & \\
\hline 37.5 Gy in 15 fractions & - & - & 14 & 14 & \\
\hline 42.5 Gy in 17 fractions & - & - & 1 & 1 & \\
\hline 40 Gy in 16 fractions & - & - & 1 & 1 & \\
\hline 36.6 Gy in 17 fractions & - & - & 1 & 1 & \\
\hline ADT & & & & & $<0.001$ \\
\hline Yes & 0 & 1 & 23 & 24 & \\
\hline No & 35 & 74 & 32 & 141 & \\
\hline
\end{tabular}

$L D R$-low-dose-rate, HDR - high-dose-rate, EBRT-external beam radiation therapy, ADT-adjuvant treatment 
was 19.5 months (range, 1.1-51.97 months). The median age at treatment was 66 years (range, $48-87$ years). A majority of patients had cT1c disease $(n=132)$, and the most prevalent Gleason score was $3+4(n=86) .85 .5 \%$ of patients had a pre-treatment PSA of $<10$. Patient characteristics are presented in Table 1.

\section{Urinary function}

AUA score was measured at the listed time points and increased for all groups after intervention. Peak scores for all groups occurred at $\leq 2$ months before improving (Figure 1). AUA score was significantly associated with time from treatment (longitudinal effect, $p<0.001$ ). AUA score was significantly higher for the LDR group at $\leq 2$ months $(p<0.0001)$ compared to the HDR monotherapy and HDR boost groups. Scores were not significantly different between the 3 groups at other time points. For

A

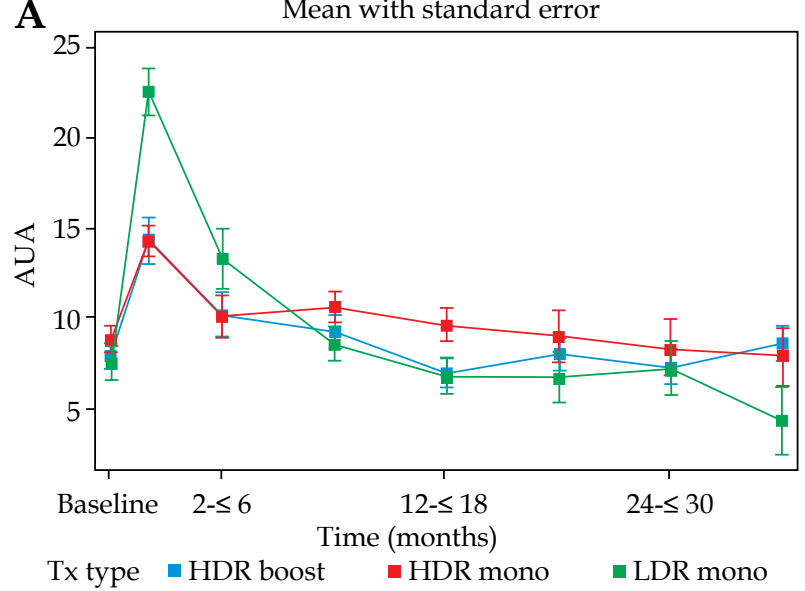

each group, the AUA score at baseline was not significantly different from the AUA score at the $>30$-month time point (all $p \geq 0.3$ ). Irr/obs urinary symptoms were also significantly associated with time from intervention for all groups (longitudinal effect, $p<0.0001$ ), with a peak incidence at $\leq 2$ months for all groups before improving (Figure 1). Irr/obs symptoms were significantly increased for the LDR group at the $\leq 2$-month time point $(p<0.0001)$ compared to the other 2 groups but were otherwise not significantly different between the 3 groups. For all groups, irr/obs scores were not significantly different from baseline at the $>30$-month time point (all $p \geq 0.45$ ). Incon scores peaked in the LDR group at $\leq 2$ months, in the HDR mono group at $>30$ months, and in the HDR boost group at 2 to $\leq 6$ months (Figure 1). Rates of incon were significantly worse in the LDR group at $\leq 2$ months compared to the other groups $(p=0.043)$.

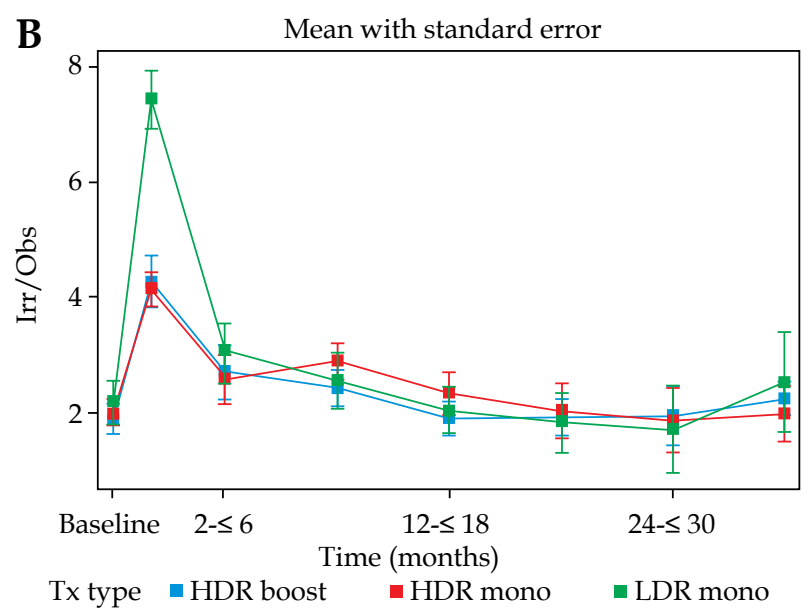

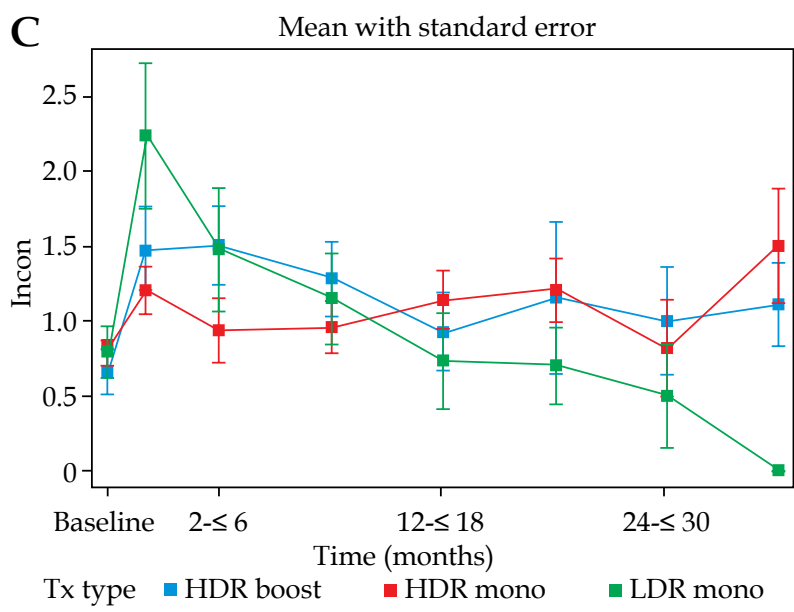

Patient survey completion rates

\begin{tabular}{|l|c|c|c|c|c|c|c|c|}
\hline $\begin{array}{l}\text { Number of patients } \\
\text { who completed the } \\
\text { survey }\end{array}$ & Baseline & $\leq 2$ months & $2-\leq 6$ & $6-\leq 12$ & $12-\leq 18$ & $18-\leq 24$ & $24-\leq 30$ & $>30$ \\
\hline LDR mono & 35 & 32 & 22 & 27 & 15 & 10 & 6 & 4 \\
\hline HDR mono & 76 & 61 & 31 & 53 & 36 & 19 & 11 & 17 \\
\hline HDR boost & 53 & 38 & 28 & 43 & 28 & 19 & 11 & 20 \\
\hline
\end{tabular}

Fig. 1. Average urinary function symptom scores over time by group. A) AUASS score; B) Irr/obs score; C) Incon score 
Incon was not significantly associated with time from intervention (longitudinal effect, $p=0.242$ ). Compared to baseline, incon scores at $>30$ months were not significantly different in the HDR boost and LDR groups. While the difference did reach significance in the HDR mono group $(p=0.044)$, the absolute difference was small, with average scores of $0.84 \pm 1.12$ and $1.50 \pm 1.62$ at baseline and $>30$ months, respectively.

\section{Bowel function}

Bowel toxicity peaked for all groups at $\leq 2$ months from intervention before improving and was significantly worse in the LDR group compared to the HDR monotherapy and boost groups at the same time point $(p=0.005)$ (Figure 2). Bowel toxicity was not significantly associated with time from intervention (longitudinal effect, $p=0.629$ ). In addition, EPIC-CP bowel toxicity score was not significantly different from baseline at $>30$ months for any group (all $p \geq 0.09$ ).

\section{Sexual function}

Rates of sexual dysfunction were not significantly associated with time from intervention (longitudinal effect, $p=0.160$ ) and did not significantly differ between the 3 groups at any time point (Figure 2). Compared to

A

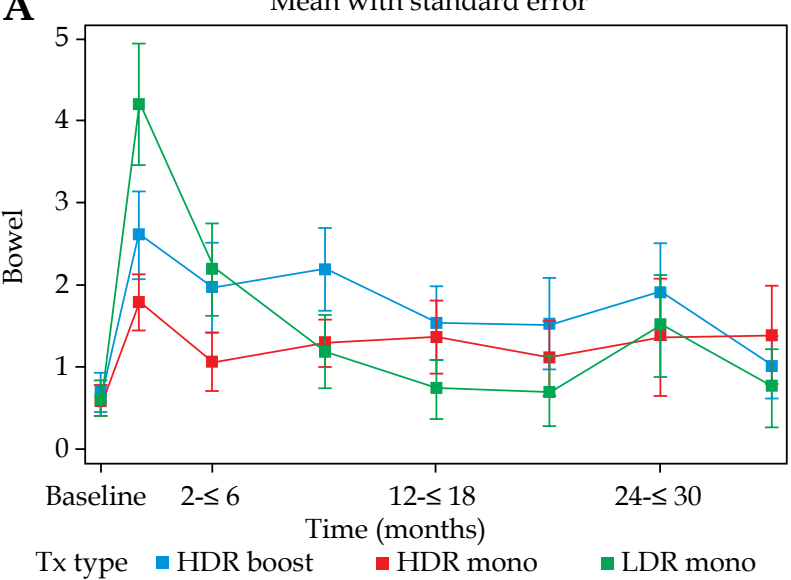

C

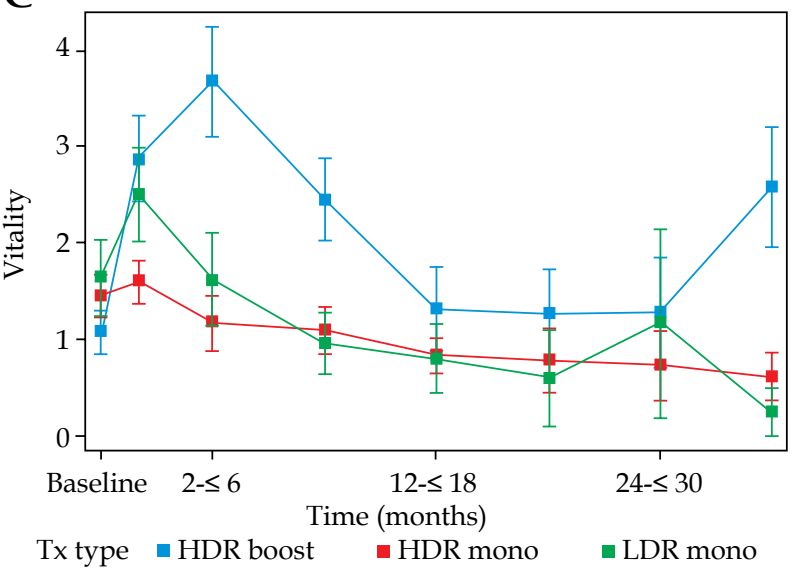

baseline, sexual function scores were significantly worse at the $>30$-month time point in the HDR boost and HDR mono groups (both $p \leq 0.001$ ). While numerically worse in the LDR group, this did not reach significance $(p=0.7)$.

\section{Vitality/quality of life}

Vitality scores were significantly worse in the HDR boost group at $\leq 2,2$ to $\leq 6,6$ to $\leq 12$, and $>30$ months $(p=0.024, p=0.0003, p=0.004$, and $p=0.010$, respectively) compared to the HDR mono and LDR mono groups. Vitality scores did significantly change with time $(p=0.0006)$ (Figure 2). In the HDR boost group, the vitality score at $>30$ months was significantly worse than at baseline $(p=0.007)$. Analysis of QOL showed peaked scores for all groups at $\leq 2$ months (Figure 2). QOL was significantly worse for the LDR group at this time point $(p=0.021)$. Scores were not significantly associated with time from treatment. Overall QOL score was significantly worse at the $>30$-month time point compared to baseline in the 2 HDR groups (for both, $p<0.03$ ), but did not reach significance in the LDR group $(p=0.85)$.

\section{Discussion}

In our study, all major functional quality of life domains were affected after treatment with brachytherapy
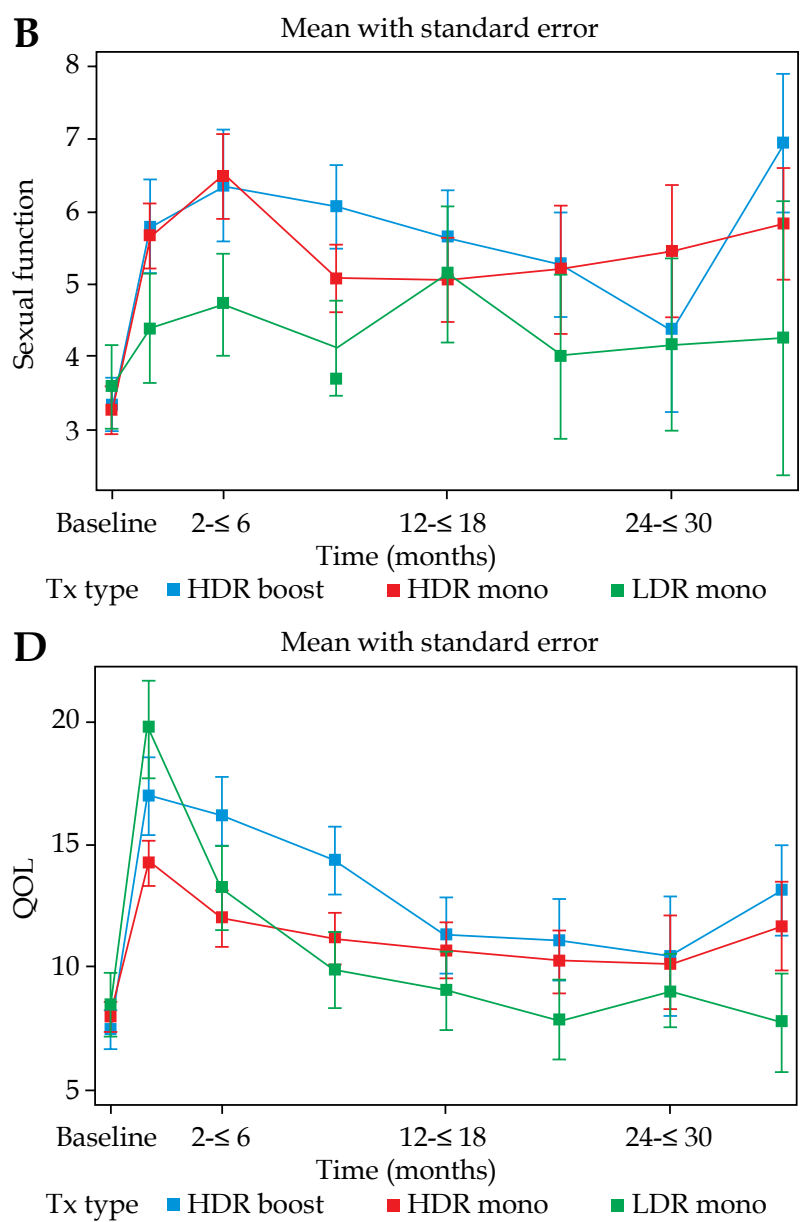

Fig. 2. Average bowel, sexual function, vitality, and QOL symptom scores over time by group. A) Bowel score; B) Sexual function score; C) Vitality score; D) Overall EPIC-CP QOL score 
for localized prostate cancer, including urinary, bowel, and sexual function, along with vitality. A majority of domains improved over time, with the exception of sexual function scores for all groups and urinary incontinence scores for the HDR mono group. Patients treated with LDR did have higher AUA, irr/obs, incontinence, bowel, and QOL scores acutely compared to the HDR and HDR + boost groups. Vitality scores were significantly worse in the HDR boost group both acutely and at the $>30$-month time point. However, the HDR boost group did have more patients who received androgen deprivation therapy (ADT), which likely contributed to this result (Table 1 ).

There have been 2 recent studies comparing HDR and LDR brachytherapy. In a single-institution retrospective study, Grills et al. compared toxicity between HDR and LDR monotherapy [16]. HDR was delivered in 4 fractions, 2 times a day over 2 days. The LDR dose was $120 \mathrm{~Gy}$. The HDR group experienced decreased rates of acute grade 1 to 3 dysuria, urinary frequency/urgency, and rectal pain, which is consistent with our data. In addition, the HDR group had lower rates of erectile dysfunction at 3 years after treatment. A surveillance, epidemiology, and end results program (SEER) analysis comparing LDR monotherapy, HDR monotherapy, EBRT + LDR boost, and EBRT + HDR boost was reported by Tward et al. [17]. Cumulative urinary adverse events were the highest in the EBRT + HDR boost group and lowest in the LDR group at 8 years. The risk of development of adverse treatment-related urinary events was highest in the 2 years after treatment for the LDR, EBRT + LDR, and EBRT + HDR groups before declining. The risk was greatest in the first 4 years for the HDR monotherapy group before declining. This can perhaps be applied to our finding of peak incontinence score in the HDR mono group at $>30$ months.

Previous single institution series have shown that grade 3 and 4 toxicity is rare with both HDR and LDR brachytherapy [30,31,32,33,34,35]. In addition, Sanda et al. prospectively measured patient-reported QOL outcomes among patients undergoing radical prostatectomy, EBRT, or brachytherapy for treatment of localized prostate cancer [5]. In this study, patients in the brachytherapy group were exclusively treated with LDR isotopes. Those in the brachytherapy group had the lowest rates of erectile dysfunction but the highest rates of urinary obstruction/ irritation. Both radiotherapy groups experienced acute bowel toxicity. As in our study, both urinary and rectal symptoms did improve with time from intervention. In fact, there was no significant difference in irr/obs symptoms at $>30$ months compared to baseline. In a population-based prospective cohort study of 1,141 men, Chen et al. compared patient-reported QOL between men treated with radical prostatectomy, EBRT, and brachytherapy, and those who were elected for active surveillance [6]. In the brachytherapy group, LDR and HDR were not differentiated. Patients in the brachytherapy group had increased rates of sexual dysfunction compared to those on active surveillance at 3 and 12 months, but not at 24 months. The same trend was seen with irritation/ obstructive urinary symptoms. Those in the brachythera- py group did not have higher rectal toxicity compared to those who were elected for active surveillance.

Chen et al.'s thoughtful comparative data of QOL changes with treatment also importantly reports QOL changes relative to baseline for each modality. This allows us to give context to the magnitude of change experienced after treatment with each modality. It is important to note that changes in sexual and urinary QOL were shown to deteriorate in the active surveillance cohort over the course of the study. Finally, the changes in Chen et al.'s brachytherapy cohort were also seen in our study in similar magnitude. We feel it is important to put into context and acknowledge that there are more QOL changes associated with multimodality therapy (HDR boost in our study), and that these are necessary for appropriate risk group adjusted treatment. HDR as a boost and ADT are reserved for our highest risk, yet still localized patients. Our data revealed multimodality therapy may have more treatment-related side effects (notably significantly worse vitality scores), yet we note this is consistent with other studies of multimodality care in the treatment of prostate cancer. For instance, the use of definitive radiotherapy with dose escalation, treatment of elective lymph nodes, and with ADT, and the addition of EBRT after prostatectomy are beneficial, well proven interventions $[5,6,36]$. Thus, we accept the relative causative QOL changes, if present, when treating with HDR boost as well. In addition, absolute changes in QOL scores were small in our study.

Limitations to our study include its retrospective nature and limited follow-up. Furthermore, patients were treated by a single brachytherapist, which may limit application to the general population. Still, compared to the above studies, ours is the first comprehensive QOL analysis comparing HDR monotherapy, LDR monotherapy, and EBRT + HDR boost in the modern era. Also, follow-up in other patient-reported QOL studies is similar to our own. For instance, Sanda et al. report 24-month follow-up in 730 of the $>1,200$ patient enrolled [5]. Thus, our study continues to be unique in its report of patient-reported QOL outcomes. As it is unlikely a randomized trial will be conducted comparing LDR and HDR brachytherapy for localized prostate cancer, retrospective reviews are used to inform decision making. LDR is the most widely employed technique in the United States given the long follow-up data, acceptable toxicity rates, and convenient delivery in one procedure. However, HDR brachytherapy better lends itself to dose escalation given the flexibility in treatment planning afforded by CT-based post-implant dosimetry. In addition, there are now multiple studies with long-term follow-up published, giving HDR a place as one of the primary treatment modalities for localized prostate cancer [31,32]. More convenient fractionation schedules for HDR have also been studied and are widely used in practice $[25,30,37]$. In our study, patients receiving HDR brachytherapy had lower acute urinary and rectal toxicity compared to the patients receiving LDR, even when combined with EBRT. However, both techniques have been shown to lead to excellent biochemical control rates, and long-term toxicity was similar in our study. 
In conclusion, given the low rates of toxicity associated with LDR and HDR brachytherapy, which largely improve with time from intervention, these techniques remain appropriate alternatives to prostatectomy for patients with localized prostate cancer.

\section{Disclosure}

Authors report no conflict of interest.

\section{References}

1. Siegel RL, Miller KD, Jemal A. Cancer Statistics, 2017. CA Cancer J Clin 2017; 67: 7-30.

2. Schröder FH, Hugosson J, Roobol MJ et al. Screening and prostate-cancer mortality in a randomized European study. N Engl J Med 2009; 360: 1320-1328.

3. Andriole GL, Crawford ED, Grubb RL 3rd et al. Mortality results from a randomized prostate-cancer screening trial. N Engl J Med 2009; 360: 1310-1319.

4. Hamdy FC, Donovan JL, Lane JA et al. 10-year outcomes after monitoring, surgery, or radiotherapy for localized prostate cancer. N Engl J Med 2016; 375: 1415-1424.

5. Sanda MG, Dunn RL, Michalski J et al. Quality of life and satisfaction with outcome among prostate-cancer survivors. N Engl J Med 2008; 358: 1250-1261.

6. Chen RC, Basak R, Meyer AM et al. Association between choice of radical prostatectomy, external beam radiotherapy, brachytherapy, or active surveillance and patient-reported quality of life among men with localized prostate cancer. JAMA 2017; 317: 1141-1150

7. Kuban DA, Tucker SL, Dong L et al. Long-term results of the M. D. Anderson randomized dose-escalation trial for prostate cancer. Int I Radiat Oncol Biol Phys 2008; 70: 67-74.

8. Zietman AL, Bae K, Slater JD et al. Randomized trial comparing conventional-dose with high-dose conformal radiation therapy in early-stage adenocarcinoma of the prostate: longterm results from proton radiation oncology group/American college of radiology 95-09. J Clin Oncol 2010; 28: 1106-1111.

9. Dearnaley DP, Sydes MR, Graham JD et al. Escalated-dose versus standard-dose conformal radiotherapy in prostate cancer: first results from the MRC RT01 randomised controlled trial. Lancet Oncol 2007; 8: 475-487.

10. Peeters ST, Heemsbergen WD, Koper PC et al. Dose-response in radiotherapy for localized prostate cancer: results of the Dutch multicenter randomized phase III trial comparing 68 Gy of radiotherapy with 78 Gy. J Clin Oncol 2006; 24: 1990-1996.

11. Beckendorf V, Guerif S, Le Prisé E et al. 70 Gy versus 80 Gy in localized prostate cancer: 5-year results of GETUG 06 randomized trial. Int I Radiat Oncol Biol Phys 2011; 80: 1056-1063.

12. Viani GA, Stefano EJ, Afonso SL. Higher-than-conventional radiation doses in localized prostate cancer treatment: a meta-analysis of randomized, controlled trials. Int J Radiat Oncol Biol Phys 2009; 74: 1405-1418.

13. Meier RM, Bloch DA, Cotrutz $C$ et al. Multicenter trial of stereotactic body radiation therapy for low- and intermediate-risk prostate cancer: survival and toxicity endpoints. Int J Radiat Oncol Biol Phys 2018; 102: 296-303.

14. Kotecha R, Djemil T, Tendulkar RD et al. Dose-escalated stereotactic body radiation therapy for patients with intermediate- and high-risk prostate cancer: initial dosimetry analysis and patient outcomes. Int J Radiat Oncol Biol Phys 2016; 116: 960-964.

15. Katz A, Formenti SC, Kang J. Predicting biochemical disease-free survival after prostate stereotactic body radiotherapy: risk-stratification and patterns of failure. Front Oncol 2016; 6: 168
16. Grills IS, Martinez AA, Hollander M et al. High dose rate brachytherapy as prostate cancer monotherapy reduces toxicity compared to low dose rate palladium seeds. J Urol 2004; 171: 1098-1104

17. Tward JD, Jarosek S, Chu $\mathrm{H}$ et al. Time course and accumulated risk of severe urinary adverse events after high- versus low-dose-rate prostate brachytherapy with or without external beam radiation therapy. Int J Radiat Oncol Biol Phys 2016; 95: 1443-1453.

18. Chao M, Spencer S, Guerrieri $\mathrm{M}$ et al. A single institution analysis of low-dose-rate brachytherapy: 5-year reported survival and late toxicity outcomes. J Contemp Brachytherapy 2018; 10: 155-161.

19. Morris WJ, Tyldesley S, Rodda S et al. Androgen Suppression Combined with Elective Nodal and Dose Escalated Radiation Therapy (the ASCENDE-RT Trial): an analysis of survival endpoints for a randomized trial comparing a lowdose-rate brachytherapy boost to a dose-escalated external beam boost for high- and intermediate-risk prostate cancer. Int J Radiat Oncol Biol Phys 2017; 98: 275-285.

20. Grimm P, Billiet I, Bostwick D et al. Comparative analysis of prostate-specific antigen free survival outcomes for patients with low, intermediate and high risk prostate cancer treatment by radical therapy. Results from the Prostate Cancer Results Study Group. BJU Int 2012; 109 (Suppl 1): 22-29.

21. Merrick GS, Tennant A, Wallner KE et al. Does supplemental external beam radiation therapy impact urinary, bowel, and erectile function following permanent prostate brachytherapy? Results of two prospective randomized trials. J Contemp Brachytherapy 2017; 9: 403-409.

22. Morgan TM, Press RH, Cutrell PK et al. Hypofractionated external beam radiation therapy in combination with HDR boost for localized prostate cancer: patient reported quality of life outcomes. J Contemp Brachytherapy 2018; 10: 211-217.

23. Barry MJ, Fowler FJ, Jr., O'Leary MP et al. The American Urological Association Symptom Index for benign prostatic hyperplasia. J Urol 2017; 197: S189-S197.

24. Chang P, Szymanski KM, Dunn RL et al. Expanded prostate cancer index composite for clinical practice: development and validation of a practical health related quality of life instrument for use in the routine clinical care of patients with prostate cancer. J Urol 2011; 186: 865-872.

25. Morton G, Loblaw A, Cheung P et al. Is single fraction 15 Gy the preferred high dose-rate brachytherapy boost dose for prostate cancer? Radiother Oncol 2011; 100: 463-467.

26. Helou J, D'Alimonte L, Loblaw A et al. High dose-rate brachytherapy boost for intermediate risk prostate cancer: Long-term outcomes of two different treatment schedules and early biochemical predictors of success. Radiother Oncol 2015; 115: 84-89.

27. Falk AT, Demontoy S, Chamorey E et al. High-dose-rate brachytherapy boost for prostate cancer: Comparison of three different fractionation schemes. Brachytherapy 2017; 6: 993-999.

28. Vigneault E, Mbodji K, Magnan S et al. High-dose-rate brachytherapy boost for prostate cancer treatment: Different combinations of hypofractionated regimens and clinical outcomes. Radiother Oncol 2017; 124: 49-55.

29. Morton G, Chung HT, McGuffin M et al. Prostate high doserate brachytherapy as monotherapy for low and intermediate risk prostate cancer: Early toxicity and quality-of life results from a randomized phase II clinical trial of one fraction of 19 Gy or two fractions of 13.5 Gy. Radiother Oncol 2017; 122: 87-92.

30. Jawad MS, Dilworth JT, Gustafson GS et al. Outcomes associated with 3 treatment schedules of high-dose-rate brachytherapy monotherapy for favorable-risk prostate cancer. Int J Radiat Oncol Biol Phys 2016; 94: 657-666. 
31. Yoshioka Y, Suzuki O, Isohashi $\mathrm{F}$ et al. High-dose-rate brachytherapy as monotherapy for intermediate- and highrisk prostate cancer: clinical results for a median 8-year follow-up. Int J Radiat Oncol Biol Phys 2016; 94: 675-682.

32. Hauswald H, Kamrava MR, Fallon JM et al. High-dose-rate monotherapy for localized prostate cancer: 10-year results. Int J Radiat Oncol Biol Phys 2016; 94: 667-674.

33. Wallner K, Roy J, Harrison L. Dosimetry guidelines to minimize urethral and rectal morbidity following transperineal I-125 prostate brachytherapy. Int J Radiat Oncol Biol Phys 1995; 32: 465-471.

34. Zelefsky MJ, Hollister T, Raben A et al. Five-year biochemical outcome and toxicity with transperineal CT-planned permanent I-125 prostate implantation for patients with localized prostate cancer. Int J Radiat Oncol Biol Phys 2000; 47: 1261-1266.

35. Beyer DC, Priestley JB, Jr. Biochemical disease-free survival following 125I prostate implantation. Int J Radiat Oncol Biol Phys 1997; 37: 559-563.

36. Moinpour CM, Hayden KA, Unger JM et al. Health-related quality of life results in pathologic stage $C$ prostate cancer from a Southwest Oncology Group trial comparing radical prostatectomy alone with radical prostatectomy plus radiation therapy. J Clin Oncol 2008; 26: 112-120.

37. Hoskin P, Rojas A, Ostler P et al. Single-dose high-dose-rate brachytherapy compared to two and three fractions for locally advanced prostate cancer. Radiother Oncol 2017; 124: 56-60. 